\title{
Quantitative disease risk scores from EHR with applications to clinical risk stratification and genetic studies
}

\author{
Danqing $\mathrm{Xu}^{1}$, Chen Wang $\mathbb{1}^{1,2,12}$, Atlas Khan $\mathbb{D}^{2,12}$, Ning Shang $\mathbb{D}^{2}$, Zihuai He ${ }^{3,4}$, Adam Gordon ${ }^{5}$, Iftikhar J. Kullo $\mathbb{D}^{6}$, Shawn Murphy $\mathbb{D}^{7,8}$, \\ Yizhao $\mathrm{Ni}^{9}$, Wei-Qi Wei ${ }^{10}$, Ali Gharavi $\mathbb{D}^{2}$, Krzysztof Kiryluk ${ }^{2}$, Chunhua Weng $\mathbb{D}^{11,13}$ and luliana lonita-Laza $\mathbb{D}^{1,13 凶}$
}

Labeling clinical data from electronic health records (EHR) in health systems requires extensive knowledge of human expert, and painstaking review by clinicians. Furthermore, existing phenotyping algorithms are not uniformly applied across large datasets and can suffer from inconsistencies in case definitions across different algorithms. We describe here quantitative disease risk scores based on almost unsupervised methods that require minimal input from clinicians, can be applied to large datasets, and alleviate some of the main weaknesses of existing phenotyping algorithms. We show applications to phenotypic data on approximately 100,000 individuals in eMERGE, and focus on several complex diseases, including Chronic Kidney Disease, Coronary Artery Disease, Type 2 Diabetes, Heart Failure, and a few others. We demonstrate that relative to existing approaches, the proposed methods have higher prediction accuracy, can better identify phenotypic features relevant to the disease under consideration, can perform better at clinical risk stratification, and can identify undiagnosed cases based on phenotypic features available in the EHR. Using genetic data from the eMERGE-seq panel that includes sequencing data for 109 genes on 21,363 individuals from multiple ethnicities, we also show how the new quantitative disease risk scores help improve the power of genetic association studies relative to the standard use of disease phenotypes. The results demonstrate the effectiveness of quantitative disease risk scores derived from rich phenotypic EHR databases to provide a more meaningful characterization of clinical risk for diseases of interest beyond the prevalent binary (case-control) classification.

npj Digital Medicine (2021)4:116; https://doi.org/10.1038/s41746-021-00488-3

\section{INTRODUCTION}

The increasing availability of rich phenotype data from electronic health records (EHR), such as the multicenter Electronic Medical Records and Genomics (eMERGE) network ${ }^{1,2}$, BioVU ${ }^{3}$ from Vanderbilt University, the Geisinger Health System's DiscovEHR in Pennsylvania ${ }^{4}$, the Harvard University/Partners Healthcare system i2b2 effort ${ }^{5}$, the United Kingdom Biobank (UKBB) ${ }^{6}$, and their linking to biobanks of human germline DNA samples provides great opportunities for genomic-based research ${ }^{7-9}$. However, inferring phenotypes from International Classification of Diseases (ICD) codes is not trivial, and many algorithms have already been proposed ${ }^{10}$. Although these algorithms can generate high-quality case/control labels for specific diseases, a main limitation is that they require extensive knowledge and involvement of human experts, are time-consuming, are not systematically applied, and can lead to inconsistencies of case definition for different algorithms ${ }^{11}$. Furthermore, they tend to perpetuate the view of common diseases as discrete entities rather than residing on a continuum. Indeed, there is a spectrum of any specific complex disease and whether an individual is labeled as a case can be arbitrary. We consider here an alternative view, namely that common diseases are the extreme tails of quantitative traits, and all of us are susceptible to specific diseases to a greater or lesser extent. Thinking quantitatively about common diseases could prove beneficial for genomic studies of phenotypes derived from $\mathrm{EHR}^{12,13}$.

Therefore an alternative approach to expert derived phenotype labels is to derive phenotypic risk scores that quantify the propensity of an individual to develop a disease. Recently, a method to compute phenotypic risk scores (PheRS) has been proposed in the context of rare Mendelian phenotypes ${ }^{14}$. This method is conceptually simple, and the authors have showed that it can be effective in identifying individuals with undiagnosed Mendelian phenotypes, and can pinpoint potentially relevant pathogenic mutations. However, the main disadvantage is that it requires a careful selection of phenotypic features for good performance, which limits its scalability and appeal, especially in applications to more complex diseases. Inspired by this approach, we propose to investigate almost unsupervised methods for phenotype risk prediction for more common diseases, that need minimal input from clinicians and can incorporate not only binary phenotypic features but also quantitative measurements such as laboratory values. In this paper, we have two main objectives: (1) derive quantitative disease risk scores for a given disease, and (2) integrate these newly derived continuous phenotypes with targeted sequencing data in eMERGE-seq to perform genetic association tests. As with the PheRS for Mendelian phenotypes, we demonstrate that the proposed quantitative disease risk scores are effective in pinpointing undiagnosed cases based on the

\footnotetext{
${ }^{1}$ Department of Biostatistics, Columbia University, New York, NY, USA. ${ }^{2}$ Division of Nephrology, Department of Medicine, Columbia University, New York, NY, USA. ${ }^{3}$ Department of Neurology and Neurological Sciences, Stanford University, Stanford, CA, USA. ${ }^{4}$ Quantitative Sciences Unit, Department of Medicine, Stanford University, Stanford, CA, USA. ${ }^{5}$ Department of Pharmacology and Center for Genetic Medicine, Northwestern University, Chicago, IL, USA. ${ }^{6}$ Department of Cardiovascular Medicine, Mayo Clinic, Rochester, MN, USA. ${ }^{7}$ Department of Biomedical Informatics, Harvard Medical School, Boston, MA, USA. ${ }^{8}$ Research Information Science and Computing, Mass General Brigham, Boston, MA, USA. ${ }^{9}$ Division of Biomedical Informatics, Cincinnati Children's Hospital Medical Center, Cincinnati, OH, USA. ${ }^{10}$ Department of Biomedical Informatics, Vanderbilt University, Nashville, TN, USA. ${ }^{11}$ Department of Biomedical Informatics, Columbia University, New York, NY, USA. ${ }^{12}$ These authors contributed equally: Chen Wang, Atlas Khan. ${ }^{13}$ These authors jointly

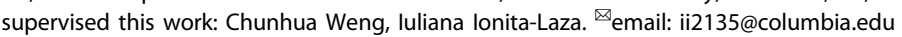


Table 1. Number of individuals (cases vs. controls) in training and test datasets.

\begin{tabular}{|c|c|c|c|c|c|}
\hline \multirow[t]{2}{*}{ Disease } & \multirow[t]{2}{*}{ \#phecodes } & \multicolumn{2}{|l|}{ Training } & \multicolumn{2}{|l|}{ Testing } \\
\hline & & Control & Case & Control & Case \\
\hline CKD & 104 & 7,334 & 17,897 & 7,334 & 17,898 \\
\hline CAD & 93 & 5,479 & 5,479 & 21,916 & 5,479 \\
\hline T2D & 132 & 3,767 & 3,767 & 9,960 & 3,768 \\
\hline $\mathrm{HF}$ & 90 & 1,724 & 1,724 & 10,806 & 1,724 \\
\hline Dementia & 143 & 879 & 879 & 7,570 & 879 \\
\hline GERD & 162 & 3,233 & 3,233 & 5,918 & 3,233 \\
\hline \multirow[t]{2}{*}{ CKD cases } & G1 & G2 & G3a & G3b & G4 \\
\hline & 2,370 & 22,204 & 6,612 & 3,332 & 1,277 \\
\hline
\end{tabular}

The number of cases at different CKD stages are also reported. The \#phecodes is the number of pre-selected phecodes used for deriving the quantitative disease risk scores for validation purposes (excludes the case defining phecodes).

phenotypic features in the EHR, and more generally in clinical risk stratification, and help improve the power of genetic association studies. Although we focus our applications on six complex traits in this paper, our methods are general and can be employed for clinical risk stratification and genetic association studies for any common disease of interest with minimal involvement of clinical expertize.

The score we propose can be viewed as a generalization of the simple PheRS score. Specifically, we develop a quantitative disease risk score as a weighted linear combination of multiple phenotypic features, these include phecodes, but can also include laboratory values, other clinical covariates, as well as natural language processing (NLP) features extracted from clinical notes when available. Our proposed approach is based on a linear combination of multiple principal components (LPC) of the phenotypic feature matrix. More details are given in the "Methods" section. We compare its performance with that of the previously proposed Phenotype Risk Score (PheRS), which combines binary phecodes in an individual ${ }^{14}$, with phecodes' weights based on the inverse prevalence of the phecode in a given population. We also include comparisons with PheNorm, a recent phenotyping algorithm that does not require expert-labeled individuals for training ${ }^{15}$.

\section{RESULTS}

\section{Description of datasets and phenotypes}

We focus here on the data from the eMERGE Network, containing 102,597 subjects, each with records in terms of ICD-9/ICD-10 codes, which the World Health Organization established to map health conditions to designated codes. In this paper we make use of phecodes, hierarchical groupings of ICD-9/ICD-10 codes, originally developed for phenome-wide association studies $\left(\right.$ PheWAS) ${ }^{16}$. The current version of phecodes (Version 1.2) has 1,866 phecodes, and 102,597 subjects in the eMERGE network have phecodes available. In addition to rich phenotypic data, 21,363 individuals have sequencing data in 109 genes chosen as part of the eMERGE specific sequencing platform ${ }^{17}$.

We describe here the datasets we used for training and testing purposes. Although we do not use a case-control label in the training, our approaches need to be trained on datasets enriched in cases for the disease under consideration, although as we explain later, only an approximate definition of case is required. That is why we refer to the proposed methods as almost unsupervised.
The Chronic Kidney Disease (CKD) case-control cohort was constructed from 98,486 subjects with available kidney function data excluding 4,111 patients with end-stage renal disease (ESRD). We considered 1,817 non-zero prevalence phecodes, which are divided into 18 categories (Supplementary Table 1). We took advantage of a CKD phenotyping algorithm recently developed within eMERGE to diagnose and place individuals on a CKD staging grid of albuminuria by estimated glomerular filtration rate $\left(\right.$ eGFR) ${ }^{18}$. Using this algorithm, the individuals were classified as having no CKD (controls) or having CKD of various severity, classified as G1, G2, G3a, G3b, and G4 stage. We divided the casecontrol dataset in Table 1 into two parts, and used $50 \%$ of the cases and $50 \%$ of controls for training purposes. We first use the entire set of phecodes to build the quantitative disease risk scores, without any pre-selection based on the disease under consideration. We compare performance with the scenario when phecodes are pre-selected based on their potential relevance to the disease of interest. Note that the selection of phecodes does not require expert knowledge, and highly automated approaches can be used for this purpose. We discuss the pre-selection of phecodes in more detail in the next section.

For consistency, we focused on the same set of 98,486 subjects as above in the analyses of additional phenotypes, including Coronary Artery Disease (CAD), Type 2 Diabetes (T2D), Heart Failure (HF), Dementia, and Gastroesophageal Reflux Disease (GERD). CAD case definition was based on a composite of myocardial infarction ${ }^{19}$. Myocardial infarction was based on selfreport or hospital admission diagnosis. This included individuals with ICD-9 codes of $410 . X, 411.0,412 . X$, or 429.79 , or ICD-10 codes of 121.X, I22.X, 123.X, 124.1, or I25.2 in hospitalization records. The case/control definitions of T2D, HF, Dementia, and GERD are based on the validated algorithms available at the Phenotype KnowledgeBase $(\mathrm{PheKB})^{20-23}$. We used $50 \%$ of the cases and an equal number of controls for training, and the rest of the cases and controls as test set for performance evaluation. The number of cases and controls for each phenotype are listed in Table 1. Those individuals who are neither case nor control for a given phenotype are treated as having unknown status.

Pre-selection of phecodes. Although our quantitative disease risk scores can be based on all available phecodes, it is of interest to compare their performance to the situation when only a preselected set of phecodes that we deem possibly relevant to the disease under consideration are included in the computation of the scores. The relevance of a phecode can be determined based on clinical expert knowledge, but this is not necessary and highly automated approaches such as associations between phecodes and polygenic risk scores (PRS) for diseases of interest can be employed. Only for the purposes of validation and assessing predictive performance, we have excluded the case defining phecodes in Supplementary Table 2 from the computations (this is valid also for the scenario when all phecodes are being used as above), except for PheNorm which uses the case defining phecodes in the training (see "Methods" section for details). For CKD, we included 104 CKD phecodes (manually selected by experts), 93 CAD phecodes (among the 'circulatory system' category, those with $p$-values $<10^{-5}$ in logistic regression of each phecode against CAD PRS), 132 T2D phecodes (manually selected by experts among the significant phecodes with $p$-values $<10^{-5}$ in logistic regression of each phecode against T2D PRS), $90 \mathrm{HF}$ phecodes (CAD feature phecodes with HF case defining phecodes removed), 143 Dementia phecodes (from the 'mental disorder' and 'neurological' categories) and 162 GERD phecodes (from the 'digestive' category). More details on the PRS calculation for the individuals in eMERGE are in the "Methods" section.

The selection of 'relevant' phecodes as described above is scalable to many common diseases given the general availability of genetic (e.g., GWAS) data for many such phenotypes. 
a

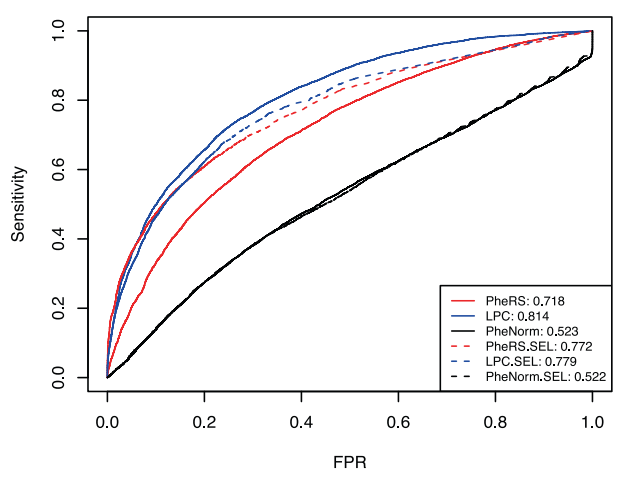

C

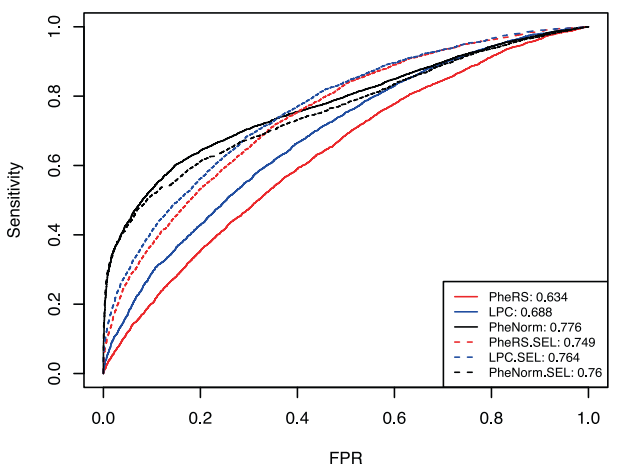

e

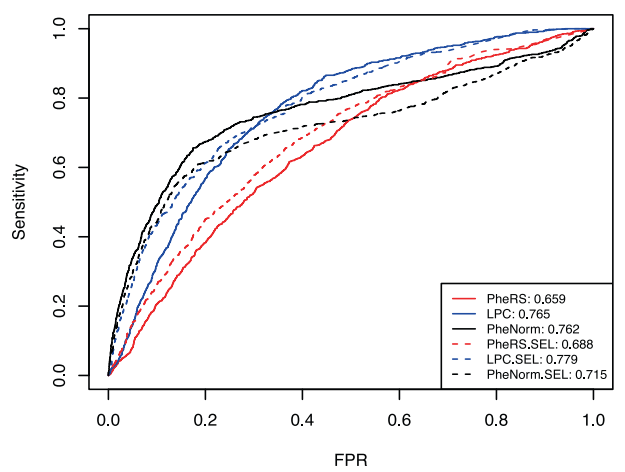

b CAD

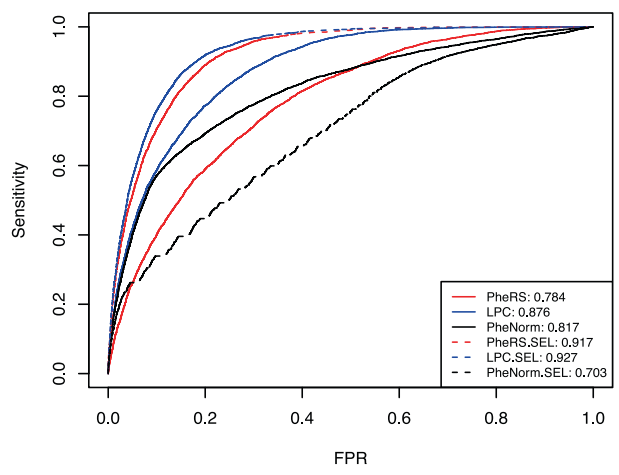

d

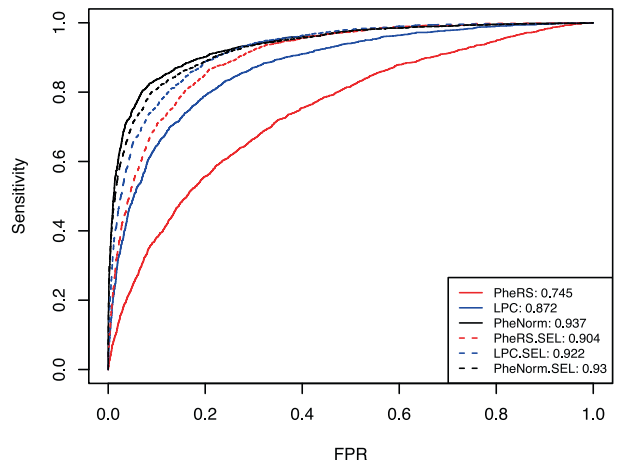

f

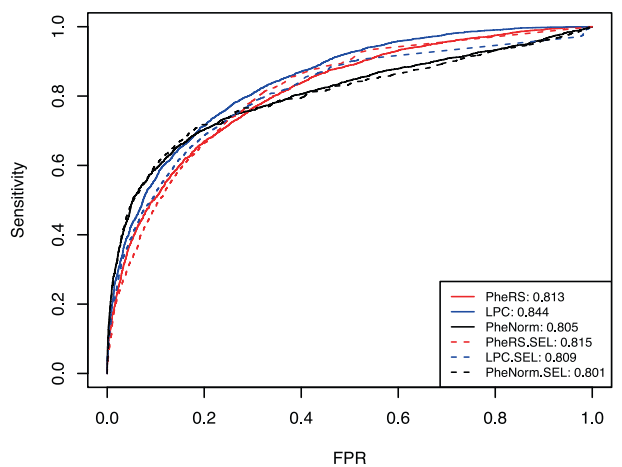

Fig. 1 ROC curves cases vs. controls for six phenotypes. ROC curves of six quantitative disease risk scores along with their AUROCs for a CKD (cases including G1, G2, G3a/b, and G4 stages), b CAD, c T2D, d HF, e Dementia, and f GERD. Quantitative disease risk scores are derived based on all phecodes (PheRS, LPC, and PheNorm), or pre-selected feature phecodes (PheRS.SEL, LPC.SEL, and PheNorm.SEL).

These existing GWAS datasets can serve as the discovery datasets for building the PRS. Then, PRS can be computed for individuals in large biobanks such as the UK biobank ${ }^{24}$, and the 'relevant' phecodes for a disease of interest can be determined based on the association between such PRS and individual phecodes. Therefore, pre-selection of phecodes using associations with PRS is possible without the need to have GWAS data for the individuals in the EHR under consideration. Nonetheless, we also provide the option to compute the quantitative disease risk scores based on all phecodes.

\section{LPC has improved prediction accuracy and robustness relative to PheRS and PheNorm}

We trained the different approaches, including PheRS, PheNorm, and LPC, on the training datasets as explained before using the same phenotypic features for the three methods (with the difference that PheNorm uses the case defining phecodes in the training, whereas LPC and PheRS do not), and then computed the quantitative disease risk scores for the individuals in the test datasets. The weights (prevalences) for the PheRS for each phenotype were estimated based on the controls in the training datasets. For the proposed LPC approach with all phecodes, the Tracy-Widom test suggested 169, 148, 131, 112, 99, and 145 significant eigenvalues (PCs) of the covariance matrix of feature phecodes for CKD, CAD, T2D, HF, Dementia, and GERD, respectively. The number of PCs reduces to $9,12,14,11,8$, and 17, with respective pre-selected phecodes for each studied phenotype. Note that the case-control labels for the individuals in the training datasets were only used in order to select the signs for the PCs in the linear combination approach, LPC (since the signs of the PCs are arbitrary). As we explain later, the choice of training set based on the gold/silver standard labels is not necessary, and weakly 
a

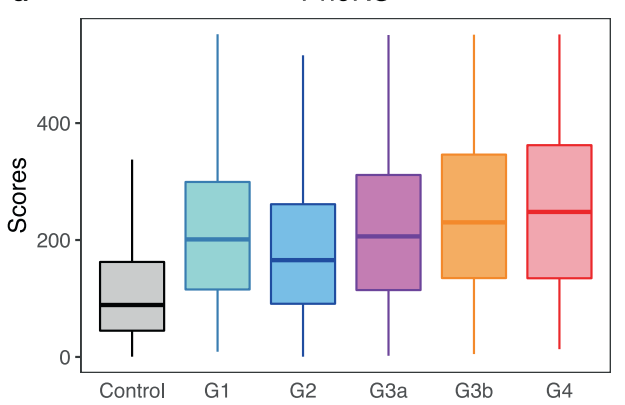

Spearman $\rho: 0.35 p$ value: $0.00 e+00$

C

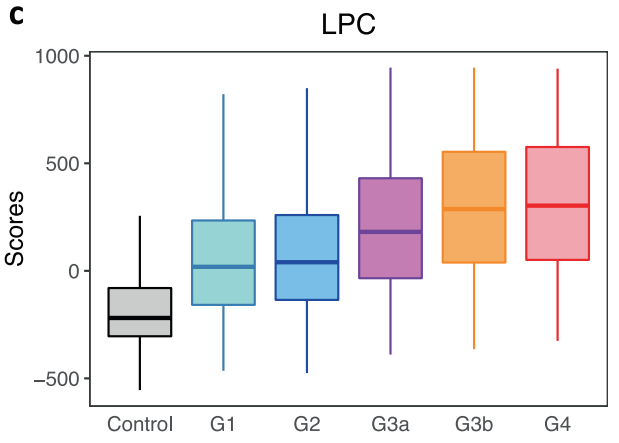

Spearman $\rho: 0.52 p$ value: $0.00 e+00$

e

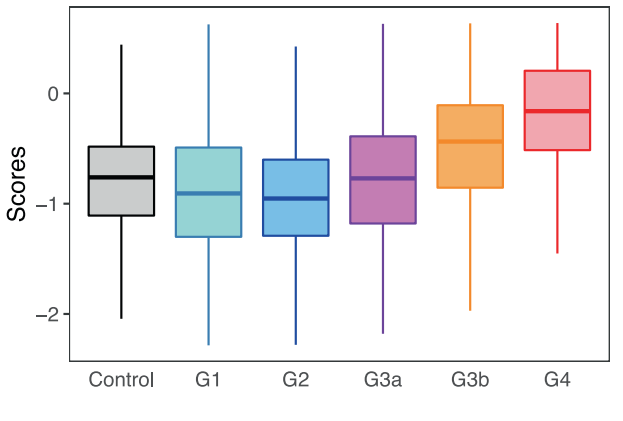

Spearman $\rho: 0.11 \mathrm{p}$ value: $1.92 \mathrm{e}-66$ b

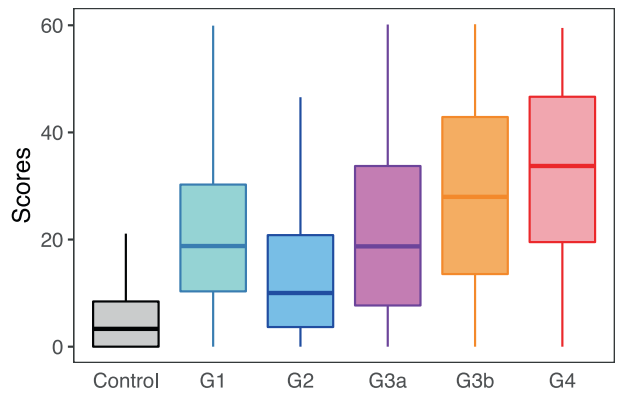

Spearman $\rho: 0.5 p$ value: $0.00 e+00$

d

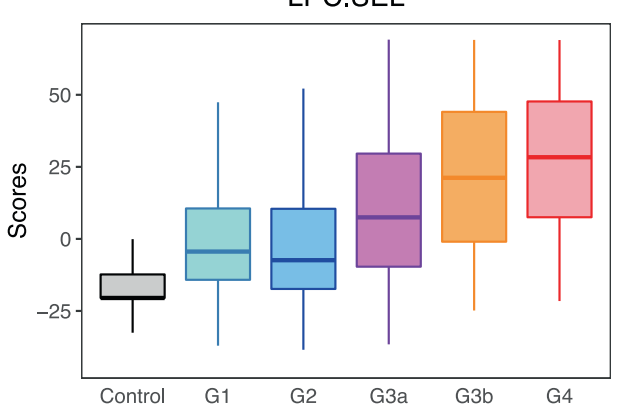

Spearman $\rho: 0.53 p$ value: $0.00 e+00$

f

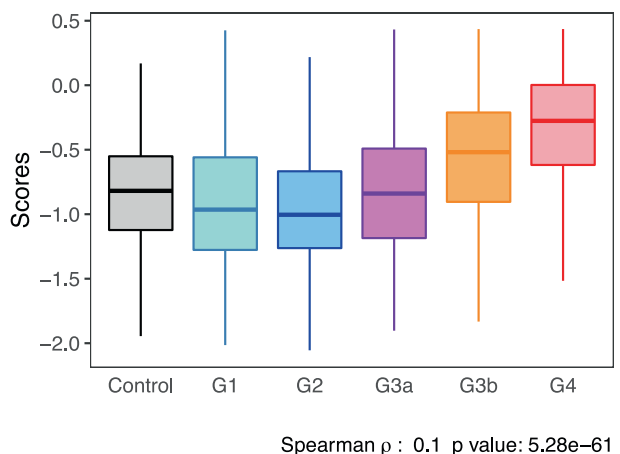

G_Staging 帛 Control 它 G2 崖 G3b

Fig. 2 Quantitative disease risk scores vs. CKD G-staging. Boxplots of quantitative disease risk scores a PheRS, b PheRS.SEL, c LPC, d LPC.SEL, e PheNorm, and f PheNorm.SEL. Quantitative disease risk scores are derived based on all phecodes (PheRS, LPC, PheNorm), or 110 preselected CKD feature phecodes (PheRS.SEL, LPC.SEL, and PheNorm.SEL). The center line, lower and upper bounds of the box represent the median, first quartile $\left(Q_{1}\right.$, or 25 th percentile), and third quartile $\left(Q_{3}\right.$, or 75 th percentile) of the data, respectively. The whisker is drawn up (down) to the largest (smallest) observed point from the data that falls within 1.5 times the interquartile range $\left(=Q_{3}-Q_{1}\right)$ above $($ below) the $Q_{3}\left(Q_{1}\right)$.

defined (soft) labels (e.g., based on presence of eGFR slope measurement for CKD, or a phecode of the target phenotype) are sufficient for training.

We compared PheRS, PheNorm, and LPC scores using either all phecodes or the pre-selected phecodes in terms of area under the receiver operating characteristic curve (AUROC) and the area under the precision-recall curve (AUPRC) in the test datasets. Note that the labels used in the performance evaluation are derived using algorithms available in PheKB rather than gold-standard labels from chart review. We acknowledge this limitation to the results we present due to the current limited availability of such gold-standard labels for the diseases we considered. We observed that LPC with all phecodes has the largest AUROC for CKD, CAD, Dementia and GERD, and the second largest for T2D and HF compared with PheRS and
PheNorm: 0.813 vs. 0.718 vs. 0.523 for CKD, 0.876 vs. 0.784 vs. 0.817 for CAD, 0.688 vs. 0.634 vs. 0.776 for T2D, 0.872 vs. 0.745 vs. 0.937 for HF, 0.765 vs. 0.659 vs. 0.762 for Dementia, and 0.844 vs. 0.813 vs. 0.805 for GERD (Fig. 1). Although PheNorm seems to perform better for T2D and HF, it is important to note that PheNorm uses the case defining phecodes in the training stage whereas they are not included in the LPC and PheRS calculations for these prediction accuracy assessments. LPC also has the largest AUPRC for CKD, CAD, and GERD (Supplementary Fig. 1). LPC with pre-selected phecodes exhibits the largest AUROC for all phenotypes except for HF when compared with PheRS and PheNorm: 0.779 vs. 0.772 vs. 0.522 for CKD, 0.927 vs. 0.917 vs. 0.702 for $C A D, 0.764$ vs. 0.749 vs. 0.760 for T2D, 0.922 vs. 0.904 vs. 0.930 for HF, 0.779 vs. 0.688 vs. 0.715 for Dementia, and 0.809 vs. 0.815 vs. 0.801 for GERD (Fig. 1). Similar 


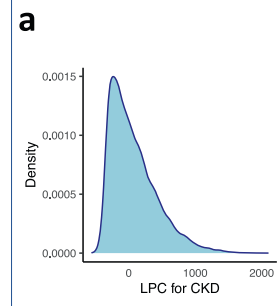

c
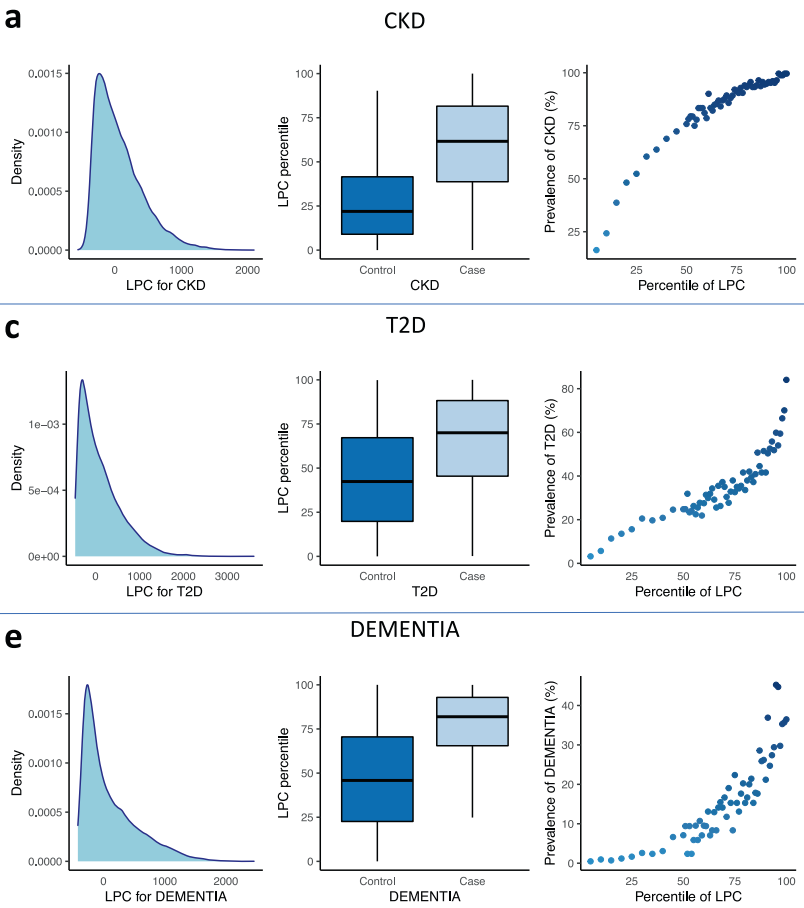

b

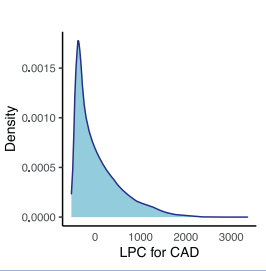

\begin{tabular}{l|l}
\hline$T 2 D$ & d
\end{tabular}
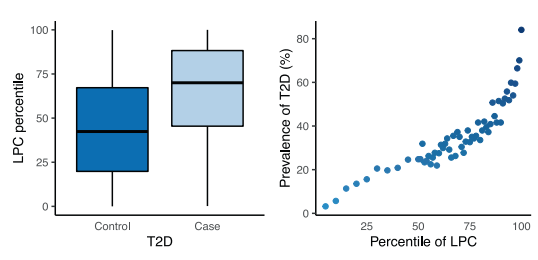

DEMENTIA
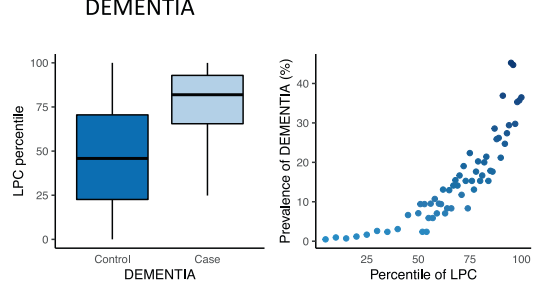
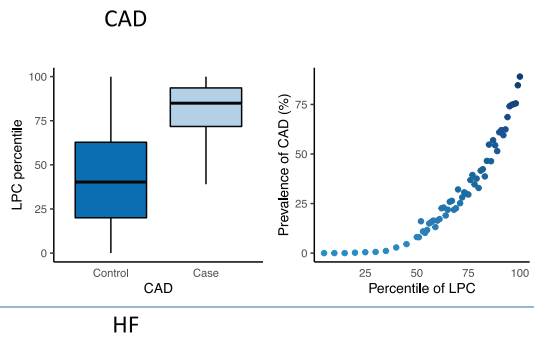

d

HF
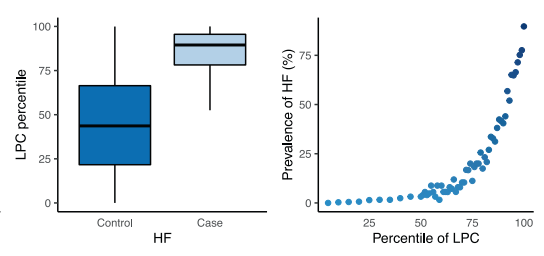

GERD
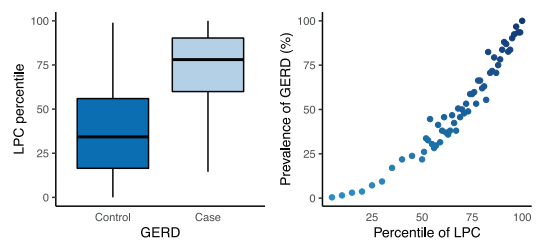

Fig. 3 Distribution of final LPC scores for six phenotypes in the test set. LPC risk scores are derived based on all phecodes. Estimated density and distribution of LPC risk scores cases vs. controls for a CKD (cases including G1, G2, G3a/b, and G4 stages), b CAD, c T2D, d HF, e Dementia, and $\mathbf{f}$ GERD. For each phenotype: left, distribution of LPC risk scores in the test set. Middle, LPC risk score percentiles among cases vs. controls. Right, case prevalence in 60 bins according to the percentiles of LPC risk scores. The center line, lower and upper bounds of the box represent the median, first quartile $\left(Q_{1}\right.$, or 25 th percentile), and third quartile $\left(Q_{3}\right.$, or 75 th percentile) of the data, respectively. The whisker is drawn up (down) to the largest (smallest) observed point from the data that falls within 1.5 times the interquartile range $\left(=Q_{3}-Q_{1}\right)$ above (below) the $Q_{3}\left(Q_{1}\right)$.

results were obtained when comparing controls with cases at different CKD G stages (Supplementary Figs. 2 and 3). Note that the AUROC values are much higher when contrasting controls with more advanced stages of CKD (G3b: 0.903 and G4: 0.908 for LPC with all other phecodes). Overall, the proposed LPC score is fairly robust to the inclusion of noisy phecodes, while PheRS can have a substantial loss in accuracy when including all phecodes. For PheNorm, pre-selecting phecodes does not in general result in performance improvement.

We observe that PheNorm has poor performance for CKD relative to other phenotypes. PheNorm score derivation relies on the count of case defining phecodes (see details in "Methods" section), so its performance depends on the correlation between the counts of case defining phecodes and PheKB derived labels. We notice high correlations between the count of case defining phecodes and case/control labels for CAD, T2D, HF, Dementia, and GERD (Spearman $\rho=0.823,0.826,0.873,0.545$, and 0.899, respectively), while the correlation for CKD is only 0.272 . This is probably due to the CKD algorithm being primarily based on lab test results. The denoising step of PheNorm regresses the normalized count of case defining phecodes on a randomly corrupted version of the features including the response variable itself and all other predictive features, with the intention to utilize the underlying association among all the features to recover the lost information of the response variable due to the random corruption. The pre-selected or all other phecodes that are included as additional features might only provide limited additional information of the presence of CKD in this denoising step, hence PheNorm may need to leverage information from features other than phecodes to better predict the CKD case/ control status.

\section{LPC improves clinical risk stratification relative to PheRS and PheNorm}

The LPC risk score correlates very well with the CKD staging (Fig. 2), providing support to the use of LPC as a measure of disease severity (note that the distributions of risk scores for CKD Control and $\mathrm{G} 1$ stages are similar since $\mathrm{G} 1$ is defined as individuals who have normal renal function but have other abnormality that makes them classified as CKD). LPC shows the strongest correlation with the CKD staging compared with PheRS and PheNorm; specifically, LPC with all phecodes has the largest Spearman's correlation coefficient $\rho$ compared with PheRS and PheNorm, $\rho=0.52(p \sim 0)$ vs. $\rho=0.35(p \sim 0)$ vs. $0.11(p=1.92 \mathrm{E}-66)$, respectively. When restricting to pre-selected phecodes, the correlation for PheRS becomes comparable to that for LPC (PheRS $\rho=0.5(p \sim 0)$ vs. LPC $\rho=0.53(p \sim 0))$.

Furthermore, we investigated the prevalence of cases in the test set among individuals at different percentiles of LPC risk score. The proportion of cases increases among the individuals with higher LPC scores, as expected (Fig. 3 and Supplementary Fig. 4). We note that the prevalence of Dementia cases among individuals with higher LPC score for Dementia is lower (29.8\% 44.7\%) than for other diseases (CKD: $98.8 \% \sim 99.6 \%$, CAD: 74.8\% 89.1\%, T2D: $54.0 \% \sim 84.0 \%$, HF: $66.4 \% \sim 89.7 \%$, GERD: $92.4 \% \sim 100 \%$ ), which may suggest that Dementia is a more difficult disease to diagnose than other diseases. The prevalence of cases among individuals with high LPC scores based on pre-selected phecodes has an overall similar or higher range compared to LPC (CKD: 97.6\% 100\%, CAD: $80.0 \% \sim 96.4 \%$, T2D: $80.3 \% \sim 98.6 \%$, HF: $84.8 \% \sim$ 93.7\%, Dementia: $52.4 \% \sim 71.8 \%$, GERD: $87.0 \% \sim 97.8 \%)$. The PheRS and PheNorm show worse performance, with lower prevalences of cases among individuals with high PheRS/ PheNorm scores relative to LPC, except for T2D and Dementia with high PheNorm scores (CKD: 92.1\% 97.6\%/71.5\% 97.6\%, 


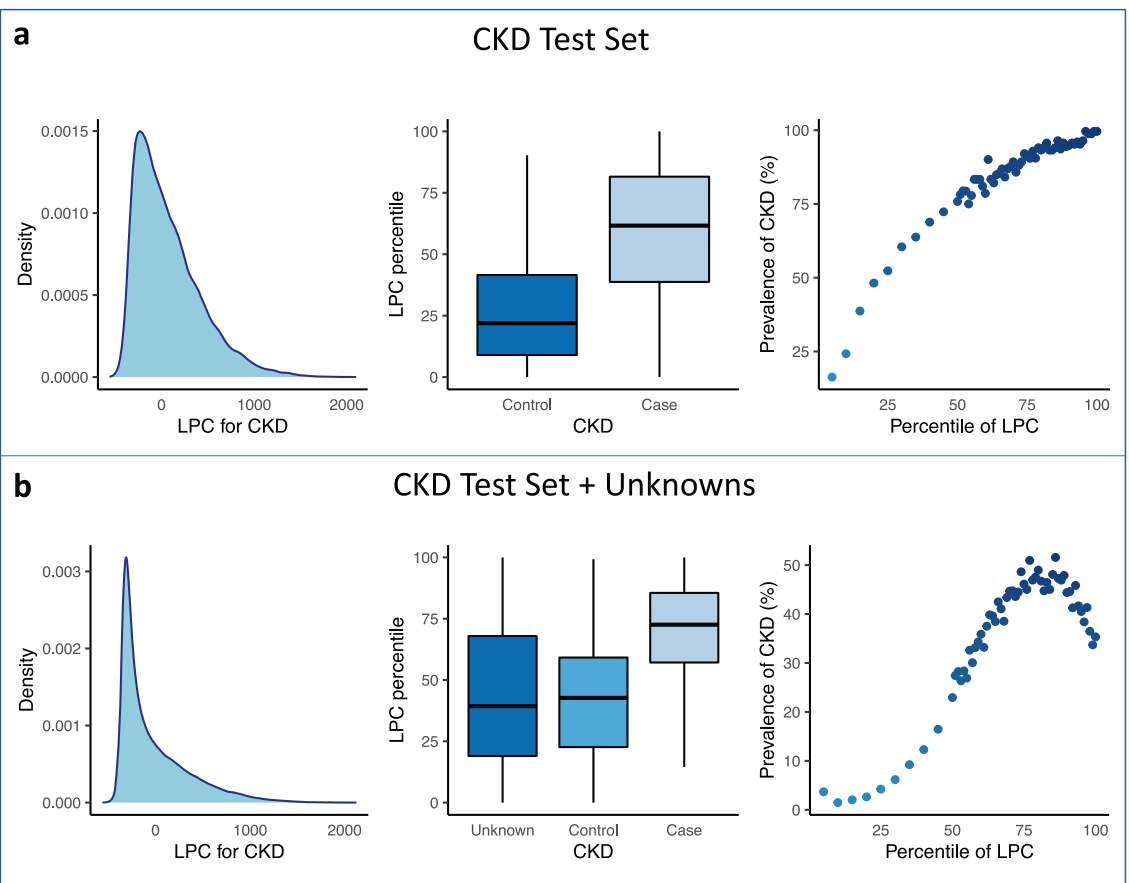

Fig. 4 Distribution of CKD LPC risk scores in the test set vs. test set + individuals with unknown status. Estimated density and distribution of LPC risk scores cases vs. controls in the $\mathbf{a}$ CKD test set and $\mathbf{b}$ with unknown status individuals added. LPC risk scores are derived based on all phecodes. Left, distribution of LPC risk scores. Middle, LPC risk score percentiles among cases vs. controls. Right, the prevalence of phenotype in 60 bins according to the percentiles of LPC risk scores. The center line, lower and upper bounds of the box represent the median, first quartile $\left(Q_{1}\right.$, or 25 th percentile), and third quartile $\left(Q_{3}\right.$, or 75 th percentile) of the data, respectively. The whisker is drawn up (down) to the largest (smallest) observed point from the data that falls within 1.5 times the interquartile range $\left(=Q_{3}-Q_{1}\right)$ above (below) the $Q_{3}\left(Q_{1}\right)$.

CAD: $51.8 \% \sim 72.2 \% / 55.8 \% \sim 86.9 \%$, T2D: $46.0 \% \sim 60.1 \% / 94.2 \% \sim$ 99.3\%, HF: $40.8 \% \sim 59.5 \% / 85.6 \% \sim 99.2 \%$, Dementia: $10.7 \% \sim$ $24.7 \% / 34.1 \% \sim 61.2 \%$, GERD: $\quad 81.5 \% \sim 97.8 \% / 91.3 \% \sim 97.8 \%)$. Restricting phenotypic features to pre-selected phecodes results in substantially higher prevalence of cases for high PheRS (CKD: 98.6\% 100\%, CAD: $83.6 \% \sim 94.5 \%$, T2D: $68.6 \% \sim 98.5 \%$, HF: 73.8\% 91.3\%, Dementia: 29.4\% 36.5\%, GERD: 83.7\% 95.7\%), but small improvement for high PheNorm (CKD: 74.6\% 76.7\%, CAD: $58.3 \% \sim 89.4 \%$, T2D: $90.5 \% \sim 99.3 \%$, HF: $79.2 \% \sim 100 \%$, Dementia: 36.5\% 55.2\%, GERD: 92.4\% 96.7\%) (Supplementary Figs. 5-8).

We have repeated the analyses above by considering a less stringent control definition, namely including in addition to controls defined by the algorithm also those with unknown status. For CKD, we have noticed an interesting pattern. For the most extreme values of the LPC quantitative disease risk scores, we noticed a sudden decrease in prevalence, suggesting that there are individuals with high quantitative disease risk scores that have unknown case status (Fig. 4). Similar results are obtained when using the other scores (PheRS, and PheNorm), with all or selected phecodes (Supplementary Figs. 9-13). This emphasizes the difficulties in obtaining accurate phenotypic labels, and the potential of the quantitative disease risk scores as discussed here to identify undiagnosed cases.

\section{LPC improves weighting of the disease-relevant phecodes relative to PheRS and PheNorm}

We show here that the weights for the disease 'relevant' phecodes are significantly higher compared with those of the rest of the phecodes (the 'irrelevant' ones) for the proposed LPC score (Table 2). The weights here are derived based on the corresponding training dataset for each disease, including all phecodes. We also show that case defining phecodes and preselected phecodes tend to have higher weights in LPC relative
Table 2. Weights for 'relevant' phecodes vs. the rest of the phecodes.

\begin{tabular}{llll}
\hline Disease & PheRS & LPC & PheNorm \\
\hline CKD & $9.82 \mathrm{E}-05$ & $1.42 \mathrm{E}-25$ & $1.36 \mathrm{E}-05$ \\
CAD & $2.41 \mathrm{E}-03$ & $2.49 \mathrm{E}-33$ & $7.36 \mathrm{E}-01$ \\
T2D & $8.10 \mathrm{E}-08$ & $3.54 \mathrm{E}-37$ & $7.79 \mathrm{E}-01$ \\
HF & $1.41 \mathrm{E}-02$ & $4.66 \mathrm{E}-17$ & $6.29 \mathrm{E}-01$ \\
Dementia & $9.38 \mathrm{E}-01$ & $1.04 \mathrm{E}-02$ & $4.02 \mathrm{E}-02$ \\
GERD & $4.15 \mathrm{E}-03$ & $1.02 \mathrm{E}-04$ & $1.54 \mathrm{E}-01$ \\
\hline
\end{tabular}

The 'relevant' phecodes include the case defining and pre-selected phecodes. Wilcoxon rank-sum test one-sided $p$-values comparing weights for 'relevant' phecodes vs. the rest of the phecodes are reported (with alternative hypothesis: 'relevant' phecodes have greater weights).

to PheRS and PheNorm (Fig. 5 and Supplementary Fig. 14). Although the PheRS weights are also significantly higher for the relevant phecodes, the $p$-values from the Wilcoxon ranksum test are much larger than those for the LPC score. PheNorm does not perform well in selecting the relevant phecodes, likely due to the approximate L2 penalty that dropout training implies ${ }^{25}$. We observe that LPC has the smallest rank-sum of pre-selected phecodes and the largest percentage of relevant phecodes among top-ranked phecodes for all phenotypes but Dementia, and the smallest rank-sum of case defining phecodes for all phenotypes except CKD (Supplementary Fig. 15). Note that the case defining phecodes have no individual weights in PheNorm, since the case defining phecodes are used together in constructing the response variable (i.e., the number of case defining phecodes) in the training component of PheNorm (see the "Methods" section for more details). 


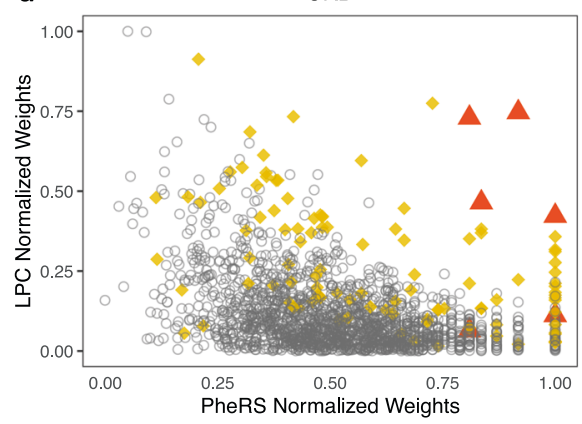

C

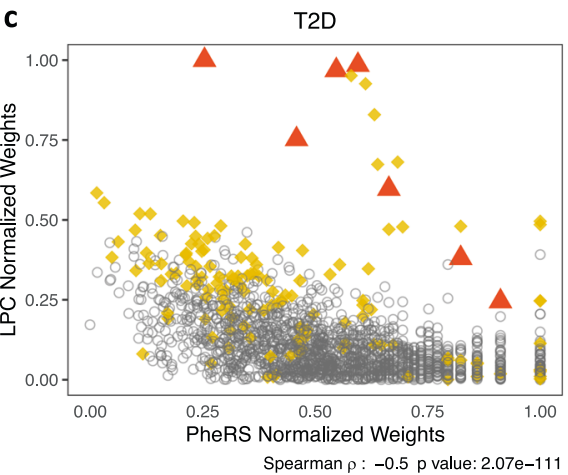

e

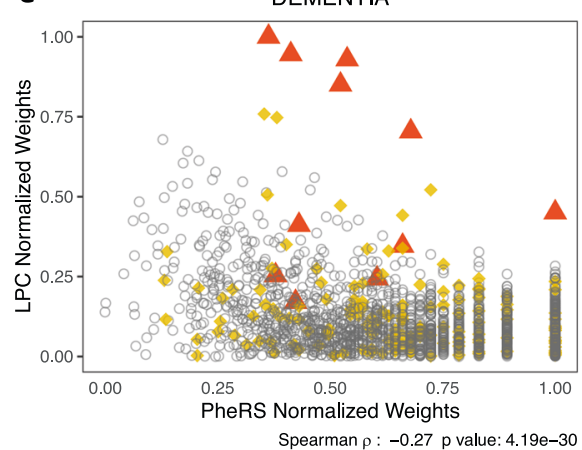

b

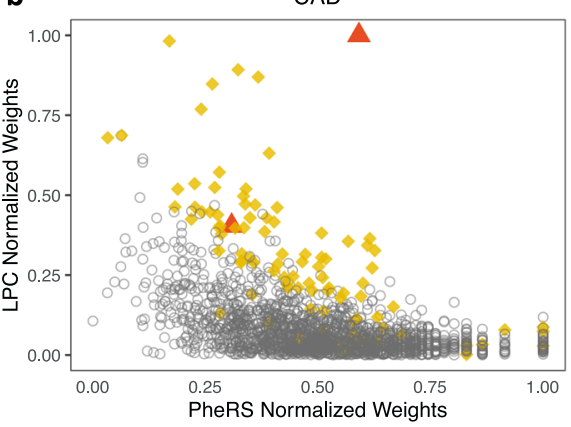

Spearman $\rho$ : -0.48 p value: $5.95 e-103$

d

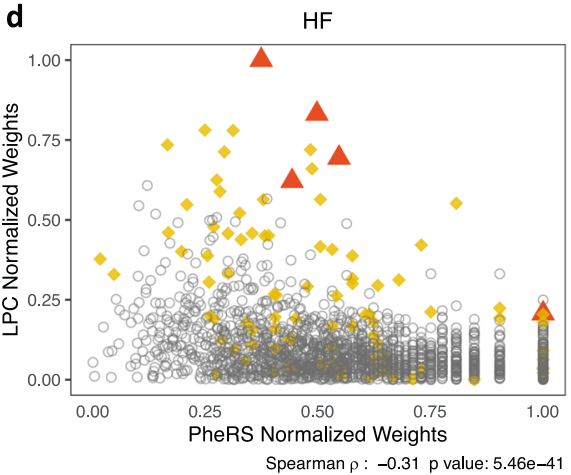

f

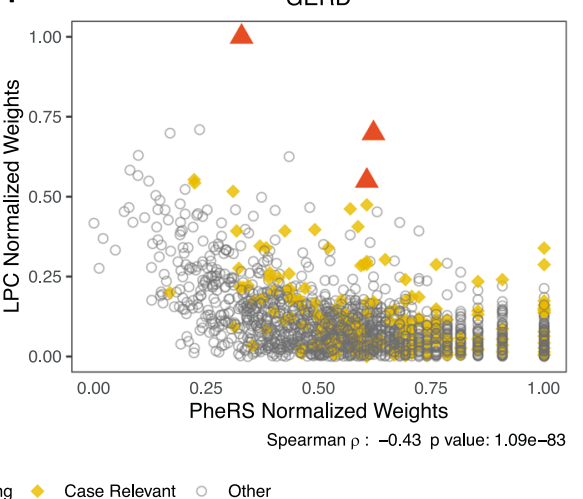

Fig. 5 Weights for all phecodes used to build PheRS and LPC for six phenotypes. Scatter plots of weights for a CKD (cases including G1, G2, $\mathrm{G} 3 \mathrm{a} / \mathrm{b}$, and G4 stages), b CAD, c T2D, d HF, e Dementia, and f GERD. The weights for the case defining and pre-selected phecodes are highlighted.

\section{Applications to genetic association studies using the eMERGE- seq dataset}

The eMERGE Network created an eMERGE specific sequencing platform and sequenced a cohort of 25,000 participants (including 14,813 Non-Hispanic White (NHW), 3,110 African American, 1,437 Asian, and 1,301 Hispanic) with an eMERGE-seq panel that includes 109 actionable genes ${ }^{17}$. The 109 genes include 56 genes from the American College of Medical Genetics and Genomics (ACMG) published a recommendation for actionable findings and additional genes deemed as potentially actionable that were selected across all eMERGE sites ${ }^{26}$. Low-quality variant calls were filtered out based on GATK recommendations, which resulted in 57,398 variants. Among the 25,000 participants, 21,363 individuals have both sequencing data and quantitative disease risk scores. More details on quality control steps are given in the "Methods" section.

We have performed comprehensive association tests with both rare and common variants within each individual gene 27,28 . For each gene, we have combined several tests, as follows:
1. Burden and dispersion tests for common and low-frequency variants (MAF > 0.01) with Beta (MAF, 1, 25) weights, where Beta $(\cdot)$ is the probability density function of the beta distribution with shape parameters 1 and 25 .

2. Burden and dispersion tests for rare variants (MAF $<0.01$ and minor allele count $(M A C) \geq 5)$ with Beta $(M A F, 1,25)$ weights.

3. Burden and dispersion tests for rare variants, weighted by functional annotations (CADD, PolyPhen).

4. Burden test for aggregation of ultra-rare variants with $M A C<$ 5 (e.g., singletons, doubletons).

5. Single variant score tests for common, low-frequency, and rare variants in the gene.

We then applied the aggregated Cauchy association test ${ }^{29}$ to combine the $p$-values from 1 to 5 to compute the final $p$-value for a gene. We adjusted for age, gender, and ten principal components of genetic variation. The distribution of LPC scores is right-skewed (Fig. 3), therefore we assumed a generalized linear model (GLM) based on the inverse-Gaussian distribution.

We focused on those rare variants that are predicted to be deleterious, as follows. First, we identified rare variants that have 

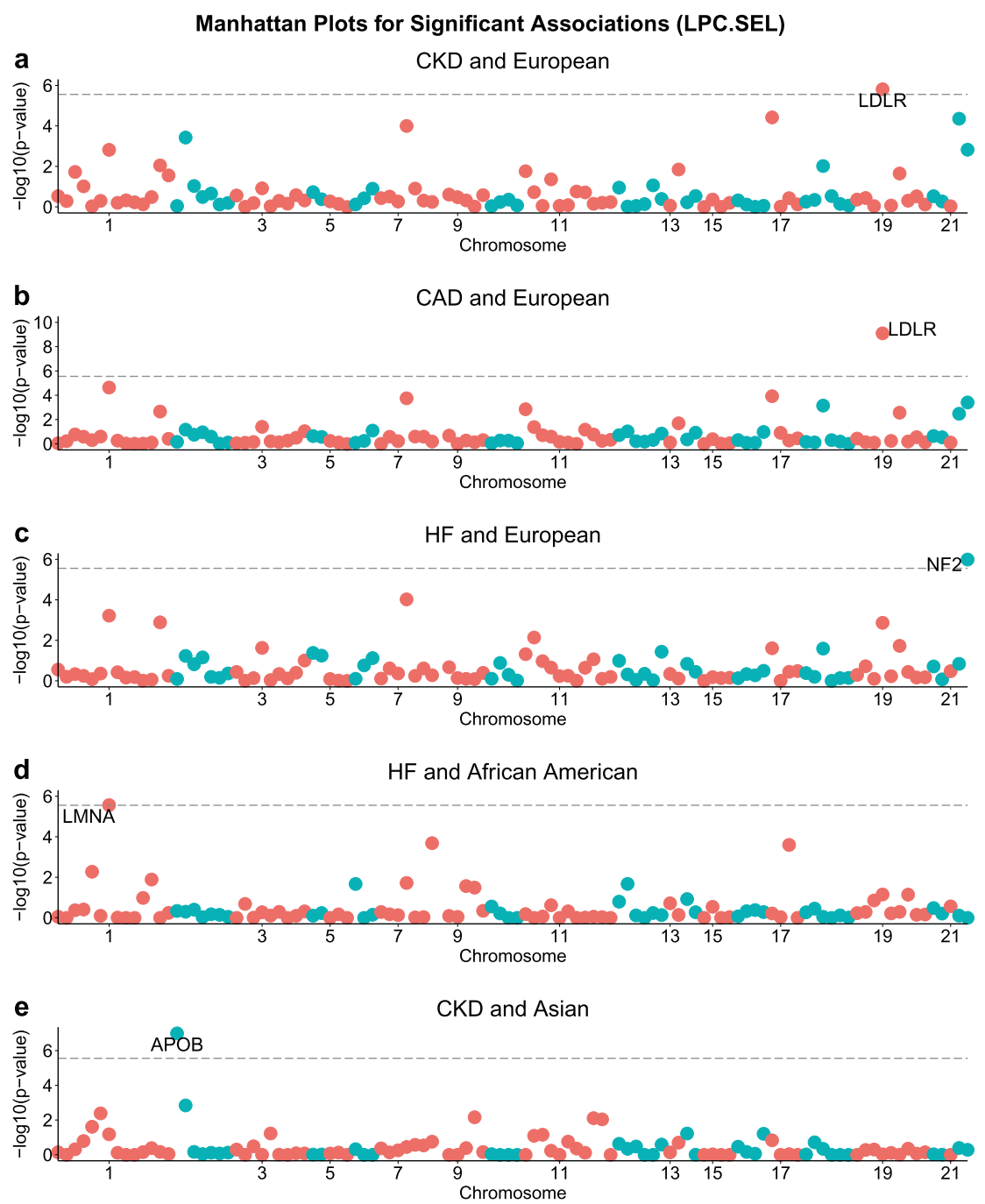

Fig. 6 Exome-wide significant gene-based test results for 107 autosomal genes on the eMERGE-seq panel using LPC with pre-selected phecodes for six diseases. Results are shown for those phenotypes and ethnic groups with at least one exome-wide significant result: a CKD and European, b CAD and European, $\mathbf{c}$ HF and European, $\mathbf{d}$ HF and African American, e CKD and Asian. The horizontal line corresponds to the exome-wide significance level.

allele frequency $<0.01$ in the Genome Aggregation Database (gnomAD v2.1.1), which integrates a large population reference cohort spanning 125,748 exome sequences ${ }^{30}$. Next, variant functional consequences were annotated using ANNOVAR $^{31}$ and RefSeq transcript data ${ }^{32}$. Moreover, the deleteriousness of proteincoding variants was assessed using PolyPhen- $2^{33}$. The final set of selected qualifying variants includes missense variants predicted as probably damaging by PolyPhen-2 and loss-of-function (LoF) variants with putative functional effects such as frameshift insertion, frameshift deletion, splicing variant, start-loss, stop-gain, and stop-loss.

We report those genes with $p$-value $<0.05 / 18000=2.78 \mathrm{E}-06$ as exome-wide significant. We focus here on the results of the LPC score using pre-selected phecodes since these scores led to more powerful genetic analyses; the genetic analyses of the quantitative disease risk scores including all phecodes is less powerful likely due to the increased environmental variation that requires increased sample sizes for improved power. Indeed no significant results are found for the LPC (Supplementary Figs. 16-19), PheRS (Supplementary Figs. 20-23), and PheNorm scores with all phecodes (Supplementary Figs. 24-27).
The significant results for LPC with pre-selected phecodes are shown in Fig. 6, and the results for the individual ethnic groups are shown in Supplementary Figs. 28-31. The associations we detected are driven by rare variants with $\mathrm{MAF}<0.01$. Indeed, for almost all significant associations the overall signal is driven by predicted deleterious variants with $\mathrm{MAF}<0.01$ and $\mathrm{MAC} \geq 5$ (Table 3 and Supplementary Table 6). The exception is LMNA and CAD, with the association driven by ultra-rare variants with $M A C<5$. We show for each significant gene the individual variants that contribute to the overall signal along with their MAC (Supplementary Figs. 33-37). The results for the two other scores PheRS and PheNorm with pre-selected phecodes are shown in Table 3, Supplementary Table 6, and Supplementary Figs. 38, 3948, and 49-52. There are no significant results for PheNorm. In contrast, several genes are exome-wide significant when using PheRS with pre-selected phecodes (Supplementary Fig. 38).

We detected significant associations between the LPC score with pre-selected phecodes for CKD and rare variants in $L D L R(p=$ $1.53 \mathrm{E}-06)$ in the European cohort. Recent large-scale multi-ethnic GWAS studies have demonstrated that $L D L R$, low-density lipoprotein receptor, is significantly associated with $C A D^{34,35}, \operatorname{LDL}^{36,37}$, 
Table 3. Genes showing significant association with at least one disease using LPC and PheRS with pre-selected phecodes.

\begin{tabular}{|c|c|c|c|c|c|}
\hline Ethnicity & Disease & Gene & p.all & p.common & p.rare \\
\hline \multicolumn{6}{|c|}{ LPC with pre-selected phecodes } \\
\hline European & CKD & LDLR & $1.530 \mathrm{E}-06$ & NA & $1.530 \mathrm{E}-06$ \\
\hline European & $\mathrm{HF}$ & NF2 & $1.009 \mathrm{E}-06$ & NA & $1.009 \mathrm{E}-06$ \\
\hline African American & $\mathrm{HF}$ & LMNA & $2.680 \mathrm{E}-06$ & NA & $2.680 \mathrm{E}-06$ \\
\hline European & CKD & CHEK2 & $4.128 \mathrm{E}-07$ & NA & $4.128 \mathrm{E}-07$ \\
\hline European & CAD & LDLR & $5.816 \mathrm{E}-07$ & NA & $5.816 \mathrm{E}-07$ \\
\hline European & CAD & CFTR & $1.266 \mathrm{E}-06$ & NA & $1.266 \mathrm{E}-06$ \\
\hline European & $\mathrm{HF}$ & CFTR & $1.189 \mathrm{E}-06$ & NA & $1.189 \mathrm{E}-06$ \\
\hline European & $\mathrm{HF}$ & LDLR & $2.306 \mathrm{E}-06$ & NA & $2.306 \mathrm{E}-06$ \\
\hline
\end{tabular}

and total cholesterol ${ }^{36,37}$. Similarly, associations with rare variants in $L D L R$ were also identified in whole-exome sequencing studies for LDL-C levels and risk of myocardial infarction (MI) ${ }^{38-40}$. Furthermore, mutations that affect $A P O E$ binding to $\mathrm{LDL}$ receptors (LDLR) render renal cells to be more susceptible to glomerular injury based on animal studies ${ }^{41}$. In fact, we also detected significant associations with rare variants in $\operatorname{LDLR}(p=7.85 \mathrm{E}-10)$ and the LPC score for CAD, also within the European cohort. Rare variants in LMNA also show significant association with Heart Failure in the African American cohort $(p=2.68 \mathrm{E}-06)$. LMNA encodes the lamin proteins that compose the nuclear membrane. Mutations in LMNA can disrupt the reorganization of laminassociated chromatin domains in cardiac myocytes through altered DNA methylation and dysregulated gene expression ${ }^{42}$. Recently, several studies have observed an association between LMNA mutations and high risk of various cardiac disorders, which include dilated cardiomyopathy that reduces the heart's ability to supply blood and causes heart failure ${ }^{42-44}$. Finally, we have also detected a significant association between rare variants in NF2 and Heart Failure $(p=1.0 \mathrm{E}-06)$. NF2 encodes the neurofibromin 2 (merlin), which regulates Hippo signaling pathways in cardiomyocytes and potentially aggravates ischemia/reperfusion injury in the heart $^{45}$.

Within the Asian group, we detected significant associations of rare variants in $A P O B$ and $L P C$ score for CKD $(p=1.0 \mathrm{E}-07)$. $A P O B$ encodes the apolipoprotein $B$ that is a major protein component of LDL. Higher serum levels of apolipoprotein A1 were associated with lower prevalence of CKD and higher eGFR in two multi-ethnic populations, while a higher apolipoprotein $\mathrm{B} / \mathrm{A} 1$ ratio was significantly associated with lower eGFR ${ }^{46}$.

For the PheRS score with pre-selected phecodes, we found significant associations for CKD and rare variants in the checkpoint 2 gene (CHEK2, $p=4.13 \mathrm{E}-07)$. Variants in CHEK2 have been found to be significantly associated with eGFR in the context of hypertension ${ }^{47}$. As with $L P C$, we have detected a significant association between rare variants in $L D L R$ and CAD PheRS score with pre-selected phecodes $(p=5.82 \mathrm{E}-07)$. PheRS for Heart Failure also shows a significant association with rare variants in LDLR $(p=2.31 \mathrm{E}-06)$. Additionally, CAD and Heart Failure PheRS are significantly associated with rare variants in CFTR $(p=1.27 \mathrm{E}$ $-06, p=1.19 \mathrm{E}-06)$. (FTR was previously identified as a susceptibility locus for CAD in a European-ancestry GWAS study ${ }^{48}$.
Within the African American cohort, we also detected a significant association between GERD PheRS and rare variants in CACNA1S $(p=1.38 \mathrm{E}-06)$.

Replication of significant association findings in other ethnic groups. We investigated if the significant associations discovered in a specific ancestry could be replicated in other ancestries. We report the $p$-values of the combined tests for each of the four populations for the five significant loci detected by LPC with preselected phecodes and their associated phenotypes in Supplcase defining phecodesementary Table 4 . The significant association of $L D L R$ and CKD in the European ancestry is nominally significant $(p<0.05)$ in the African American cohort $(p=1.43 \mathrm{E}-03)$. The association between LMNA and HF discovered in the African American ancestry is replicated in the European population ( $p=$ $5.99 \mathrm{E}-04)$. Among the six significant associations found by PheRS with pre-selected phecodes, three significant associations in the European ancestry, CFTR and CAD, CFTR and HF, and LDLR and HF, are replicated in the African American population with nominal significance $(p=3.53 \mathrm{E}-02, p=1.95 \mathrm{E}-02$, and $p=4,81 \mathrm{E}-02$, respectively). These replications provide additional evidence for our genetic findings, and also reflect the increased power of the European and African American cohorts which have larger sample sizes relative to the two other ancestries.

Binary phenotypes defined based on phecodes. A common way to analyze phenotypes derived from EHR data is to use binary ICD codes for phenotypes of interest ${ }^{49}$. To compare with such a strategy, here we used the case defining phecodes (Supplementary Table 2) to identify cases and controls in each ethnic group, and then ran the same association tests as discussed above for the quantitative disease risk scores, focusing on data from Europeans since the European group is the only group with an adequate number of cases (Supplementary Table 5). Results are shown in Supplementary Fig. 53. We have not detected any significant associations between rare variants in 107 autosomal genes and the binary phenotypes, in contrast to several associations we have identified with the quantitative disease risk scores, as described above. This comparison with results from binary phecode-defined phenotypes illustrates the potential benefit for genetic association studies when using quantitative disease risk scores. 


\section{DISCUSSION}

We have proposed an almost unsupervised method to derive a quantitative disease risk score, LPC, based on phenotypic features available in EHR from health systems. The proposed quantitative disease risk score has several advantages: (1) it can be derived on a large number of individuals using only minimal clinical input, (2) it can be derived with only weak labels, as opposed to limited gold/silver standard label information that may be available in the EHR, (3) it can help stratify individuals according to disease risk severity, and identify undiagnosed cases, (4) it can identify disease-relevant features, and (5) it can take advantage of biobanks linked to clinical information from EHR to perform potentially more powerful genetic association studies. Beyond these advantages, LPC, as a quantitative disease risk score, represents a different, promising direction in deriving phenotypes from EHR features, that can provide a more meaningful characterization of clinical risk for diseases of interest beyond the prevalent binary (case-control) classification.

Although we have focused here on using structured data such as phecodes, it is important to supplement these with other unstructured data including NLP features extracted from clinical notes, health information generated via mobile health devices, etc. to improve prediction accuracy. The proposed score can easily incorporate such features when available. In our limited analyses, incorporating lab measurements for CKD did not significantly improve the prediction accuracy. A possible reason may be that the presence of lab measurements is correlated to the presence of relevant phecodes/symptoms and that decreases the potential benefit from including them (Supplementary Fig. 54).

The different quantitative disease risk scores can be regarded as weighted linear combinations of phenotypic features, with different ways to derive the weights. The weighting scheme is particularly important when all phecodes are included, and as we have shown the proposed LPC score can upweight the diseaserelevant phecodes, leading to higher accuracy relative to the comparison scores, PheRS and PheNorm. However, when the phecodes are pre-selected according to their potential relevance for the disease under investigation, the weighting strategy is less important, as expected.

The proposed score is easy to compute using features available in the EHR, is scalable to many diseases, and is almost unsupervised. This is important because labeling clinical data requires detailed knowledge from human experts, and can be time-consuming. Although LPC does not require explicit labels in the training stage, it does require some knowledge of case status, so training sets can be enriched in cases. An alternative to highly accurate labels are labels derived from simple filters, such as at least one relevant ICD-9 code for the phenotype of interest (or similar silver standard labels). We explored the performance when using such different training sets, and found minimal effect on the performance. Another important advantage of the proposed quantitative score is that it can serve as a measure of disease severity, and therefore helps identify individuals with higher severity, and even individuals without a clinical diagnosis. In particular, for CKD we have shown that LPC correlates very well with the CKD staging by estimated glomerular filtration rate (eGFR) and even helps identify individuals with very high LPC scores which are missed by the phenotyping algorithm because they miss laboratory-based eGFR measurement.

We have performed genetic associations with the derived quantitative disease risk scores, using targeted sequencing data from eMERGE-seq, and have shown that we can identify several significant associations with variants in potentially relevant genes for the diseases considered here. In contrast, using phecodes to define binary phenotypes as commonly done in genetic association studies based on EHR phenotypes has led to no significant genes in Europeans, the group with the largest number of cases for the phenotypes considered here, highlighting the increase in power afforded by quantitative disease risk scores. The availability of large EHR systems linked to biobanks such as the eMERGE and UK Biobank opens up the possibility to perform genetic associations with a large number of phenotypes genome-wide. One of the issues recently recognized in such studies is the potential for highly imbalanced datasets where the number of controls can be much higher than the number of cases for a particular phenotype ${ }^{49}$; conventional association tests, which do not adjust for case-control imbalance can lead to biased results and increases in false positives. The proposed approach by deriving quantitative disease risk scores for all individuals alleviates this bias.

In summary, we propose almost unsupervised methods to derive quantitative disease risk scores from information in EHR that require minimal input from domain experts, and that can improve the utility of using such EHR-derived phenotypes in genomics research. Future work in developing new quantitative disease risk scores could be beneficial for leveraging the rich phenotypic information in EHR.

\section{METHODS \\ Notations}

Suppose we have $J$ phenotypic features for a given set of $n$ subjects; these include phecodes, but can also include laboratory values and other clinical covariates. Each phenotypic feature is centered and scaled by its sample standard deviation in the preprocessing step. The goal is, for a given (complex) disease phenotype, to construct a quantitative disease risk score (QRS) as a weighted linear combination of multiple phenotypic features, i.e., the score for subject $i$ is defined as

$\mathrm{QRS}_{i}=\sum_{j=1}^{J} w_{j} x_{i j}$

where $x_{i j}$ is the standardized value of $j$ th phenotypic feature for subject $i$. Here we consider several possible (almost) unsupervised methods to derive the set of weights $w_{j}^{\prime}$ s. We denote by $\boldsymbol{X}_{n \times J}$ the matrix of $J$ standardized phenotypic features for the $n$ subjects. Let $\boldsymbol{Q}$ be the $J \times J$ sample correlation matrix of the $J$ phenotypic features.

\section{Phenotype risk score (PheRS)}

The phenotype risk score (PheRS) has been recently proposed to combine binary phecodes in an individual ${ }^{14}$, with phecodes' weights based on the inverse prevalence of the phecode in the controls in the training dataset. Given a dataset of $N$ subjects, the weight for phenotypic feature $j$ is calculated as:

$w_{j}=\log \frac{N}{n_{j}}$,

where $n_{j}$ is the number of individuals with phenotypic feature $j$. Hence, less prevalent phenotypic features are given higher weight compared to the more common ones. The rationale behind this weighting scheme is that lower frequency phecodes are more likely to be related to the risk of disease in general, an assumption that may be reasonable. However, these weights are not related to the phenotype under consideration.

For subject $i$, the PheRS for a specific disease is calculated as in $^{14}$ :

$\mathrm{PheRS}_{i}=\sum_{j=1}^{J} w_{j} \mathbb{1}_{\{\text {subjectihasphenotypicfeaturej }\}}$.

\section{PheNorm}

PheNorm is a phenotyping algorithm that does not require expert-labeled samples for training ${ }^{15}$. PheNorm relies on automated feature curation. To make it comparable to LPC and PheRS, we only trained PheNorm using phecode features. We implemented a version of PheNorm in two steps, as follows.

In the first step, we normalize the most predictive features such as the number of ICD codes or mentions of the target phenotype to resemble a normal mixture distribution. Specifically, we denote by $x_{\text {PHECODE }}$ the 
number of case defining phecodes for a given disease. We use the health care utilization measure denoted by $x_{\mathrm{utl}}$ to normalize $x_{\mathrm{PHECODE}}$ since $x_{\text {PHECODE }}$ tends to be high for subjects with more health care utilization regardless of their true disease status; the number of distinct age at observation in ICD code history is considered in our implementation. The distribution of the normalized count of phecodes $z_{\text {PHECODE }}=\log (1+$ $\left.x_{\text {PHECODE }}\right)-a \log \left(1+x_{\mathrm{Utl}}\right)$ with an appropriate choice of $a$ is approximately a normal mixture distribution with $Z_{\mathrm{PHECODE}} \mid Y \sim N\left(\mu_{Y}, \sigma^{2}\right)$, where $Y$ is an indicator of the true disease status. The optimal value of $a$ is chosen to minimize the difference between the empirical distribution of $z$ and its normal mixture approximation (see ref. ${ }^{15}$ for more details). In the second step, we aggregate the information in the larger set of additional features (pre-selected or all other phecodes, excluding the case defining phecodes) with denoising self-regression via dropout training. The data matrix with columns of the normalized counts of case defining phecodes and set of candidate features $Z=\left[z_{\mathrm{PHECODE}}, z_{1}, \ldots, z_{p}\right]$, is randomly corrupted to obtain $\tilde{Z}$ with

$\tilde{Z}_{i j}=Z_{i j}^{W_{i j}}\left(\operatorname{Mean}\left(Z_{\mathrm{j}}\right)\right)^{1-W_{i j}}$,

where Mean $\left(Z_{j}\right)$ is the mean of the $j$ th column $Z$ and $\left\{W_{i j}\right\}$ are independent and identically distributed Bernoulli random variables with drop out rate $\mathbb{P}\left(W_{i j}=0\right)=r\left(r=0.3\right.$ in our applications). Then $Z_{\mathrm{PHECODE}}$ is predicted with $\tilde{Z}$ by ordinary least squares regression to obtain the regression coefficient vector $\boldsymbol{\beta}$. The final PheNorm score of subject $i$ can be obtained by the weighted linear combination of health care utilization and candidate feature sets, with feature vector $z_{i}$

PheNorm $i=z_{i}^{\top} \boldsymbol{\beta}$.

The actual implementation follows the scripts in R package sureLDA ${ }^{50}$, where random sampling with replacement of observations in each training set is used to form a bootstrap of size $10^{5}$.

\section{Principal component based score (LPC)}

Principal component analysis (PCA) is a standard approach to reduce the dimension of the phenotypic feature space, and identify a small number of principal components (PCs). In particular, we have the spectral decomposition of $\boldsymbol{Q}, \boldsymbol{Q}=\sum_{j=1}^{J} \lambda_{j} \boldsymbol{u}_{j} \boldsymbol{u}_{j}^{\top}$, where $\lambda_{1} \geq \cdots \geq \lambda_{j}>0$ are eigenvalues of $\boldsymbol{Q}$ and $\boldsymbol{u}_{j}$ is the $j$ th eigenvector associated with the jth largest eigenvalue.

One possible composite score is based on using the entries of the top eigenvector $\boldsymbol{u}_{1}$ (i.e., the loadings) as the weights for the $J$ phenotypic features. Beyond the first principal component, it is possible that additional principal components are also informative ${ }^{51,52}$, and so we consider combining multiple PCs as a linear combination (LPC), especially when including a large number of phenotypic features. The general form of the LPC score for subject $i$ is

$\mathrm{LPC}_{i}=\sum_{k=1}^{K} \beta_{k} \mathrm{PC}_{i k}$,

where $K \leq J$ represents the number of PCs being included in the linear combination, and $\mathbf{P C}=\mathbf{X U}$ is the $P C$ score matrix $(\boldsymbol{U}$ is the matrix of eigenvectors). $K$ can be determined using the Tracy-Widom test ${ }^{53}$, a hypothesis test to identify significant eigenvalues of the covariance matrix.

There are several considerations with the LPC method. The first is the choice of the sign of individual PCs in the linear combination, since the signs of the PCs are arbitrary. We use the training data to help us identify the sign of each PC. Namely, the sign of each PC is adjusted so that the mean PC value of "cases" is higher than that for "controls". We refer to the LPC approach as almost unsupervised, as we do use some approximate label information to determine the signs of individual PCs in the linear combination. However, we do not need to use gold or even silver standard labels, and weakly defined labels are sufficient (e.g., we could define as "cases" those individuals with some lab result present, e.g., the estimated glomerular filtration rate (eGFR) in the case of CKD). The second important consideration is the choice of weights. We choose here to use the corresponding eigenvalues $\lambda$ as weights, so higher weight is assigned to those components with higher amount of variance explained. However the lower PCs (corresponding to smaller eigenvalues) can be as useful for prediction as the top PCs, and hence the approach here may not be optimal. If some amount of labeled data is available, weights can be learned by regression models, such as principal component regression and partial least squares.

Another possible approach would be to do non-negative matrix factorization $(\mathrm{NMF})^{54}$. Unlike PCA, NMF constrains the factor loadings to be non-negative. Although we do not expect improved accuracy compared with the less constrained PCA approach, NMF can lead to better interpretability.

\section{Construction of feature matrix}

Each individual in the eMERGE network has raw longitudinal records of ICD-9 and ICD-10 codes, which can be mapped to phecodes ${ }^{55}$, and the presence of a particular phecode is defined by at least two occurrences of the corresponding ICD-9 or ICD-10 codes in individual health records. Each phecode is used as a proxy of the corresponding condition. Note that here the absence of a phecode may be due to no assessment of the condition or no record of healthy condition. The feature matrix of 98,486 individuals is centered and scaled before splitting into training and test sets for the Eigen and PC-based approaches. The phecode features are on the original scale for PheRS derivation.

\section{Polygenic risk score calculation}

We used the LDPred computational algorithm ${ }^{56}$ to derive a genome-wide polygenic risk score for CKD, CAD, and type 2 diabetes in the eMERGE cohort $(n=102,138)$. We used the optimized GWAS summary statistics (weights) for these traits from ${ }^{19,57,58}$. More specifically, we calculated the PRS by summing the genotype of each risk allele carried by an individual and weighting each variant by its natural logarithm of the relative risk extracted from the GWAS. We then performed an association between these PRS scores and each phecode in turn.

\section{Quality control of eMERGE-seq panel variants}

Quality control (QC) for the sequencing dataset is performed based on the quality metrics of variant and genotype calling according to GATK best practices recommendations ${ }^{59}$. Specifically, we filter out the low-quality SNVs with QD (quality by depth) $<2$, MQ (root mean square mapping quality) $<40$, FS (Fisher strand) $>60$, SOR (strand odds ratio) $>3$, MQRankSum (mapping quality rank-sum test) $<-12.5$, or ReadPosRankSum (read position rank-sum test) $<-8$. For indels, we exclude the variants that have $\mathrm{QD}<2$, ReadPosRankSum $<-20, \mathrm{FS}>200$, or $\mathrm{SOR}>10$. After $\mathrm{QC}$, the resulting dataset includes 57,398 variants.

\section{Ethics statement}

The study was approved by the Columbia University Institutional Review Board (IRB protocol numbers IRB-AAAP7926 and IRB-AAAO4154) and individual IRBs at all eMERGE-III network sites contributing human genetic and clinical data. All eMERGE participants provided informed consent to participate in genetic studies.

\section{Reporting summary}

Further information on research design is available in the Nature Research Reporting Summary linked to this article.

\section{DATA AVAILABILITY}

The development version of R package QDRS and documentation of quantitative disease risk scores from eMERGE dataset are available online (https://github.com/ danqingxu/QDRS). The eMERGE-III genetic datasets with linked phenotypes are accessible through dbGAP (accession number: phs001584.v1.p1).

Received: 9 March 2021; Accepted: 6 May 2021; Published online: 23 July 2021

\section{REFERENCES}

1. Gottesman, O. et al. The electronic medical records and genomics (eMERGE) network: past, present, and future. Genet. Med. 15, 761 (2013).

2. McCarty, C. A. et al. The eMERGE Network: a consortium of biorepositories linked to electronic medical records data for conducting genomic studies. BMC Med. Genomics 4, 1-11 (2011).

3. Pulley, J., Clayton, E., Bernard, G. R., Roden, D. M. \& Masys, D. R. Principles of human subjects protections applied in an opt-out, de-identified biobank. Clin. Transl. Sci. 3, 42-48 (2010). 
4. Carey, D. J. et al. The Geisinger MyCode community health initiative: an electronic health record-linked biobank for precision medicine research. Genet. Med. 18, 906 (2016).

5. Murphy, S. N., Mendis, M. E., Berkowitz, D. A., Kohane, I. \& Chueh, H. C. Integration of clinical and genetic data in the i2b2 architecture. In AMIA Annual Symposium Proceedings, Vol. 2006, 1040 (American Medical Informatics Association, 2006).

6. Bycroft, C. et al. The UK Biobank resource with deep phenotyping and genomic data. Nature 562, 203-209 (2018).

7. Abul-Husn, N. S. \& Kenny, E. E. Personalized medicine and the power of electronic health records. Cell 177, 58-69 (2019).

8. Kohane, I. S. Using electronic health records to drive discovery in disease genomics. Nat. Rev. Genet. 12, 417-428 (2011).

9. Wolford, B. N., Willer, C. J. \& Surakka, I. Electronic health records: the next wave of complex disease genetics. Hum. Mol. Genet. 27, R14-R21 (2018).

10. Kirby, J. C. et al. PheKB: a catalog and workflow for creating electronic phenotype algorithms for transportability. J. Am. Med. Inform. Assoc. 23, 1046-1052 (2016).

11. RL, R. et al. A comparison of phenotype definitions for diabetes mellitus. J. Am. Med. Inform. Assoc. 20, e319-e326 (2013).

12. Plomin, R., Haworth, C. M. \& Davis, O. S. Common disorders are quantitative traits. Nat. Rev. Genet. 10, 872-878 (2009).

13. Sinnott, J. A. et al. Improving the power of genetic association tests with imperfect phenotype derived from electronic medical records. Hum. Genet. 133, 1369-1382 (2014).

14. Bastarache, L. et al. Phenotype risk scores identify patients with unrecognized Mendelian disease patterns. Science 359, 1233-1239 (2018).

15. $\mathrm{Yu}, \mathrm{S}$. et al. Enabling phenotypic big data with phenorm. J. Am. Med. Inform. Assoc. 25, 54-60 (2018).

16. Denny, J. C. et al. Systematic comparison of phenome-wide association study of electronic medical record data and genome-wide association study data. Nat. Biotechnol. 31, 1102 (2013).

17. eMERGE Consortium. Harmonizing clinical sequencing and interpretation for the eMERGE III network. Am. J. Hum. Genet. 105, 588-605 (2019).

18. Shang, N. et al. Medical records-based chronic kidney disease phenotype for clinical care and "big data" observational and genetic studies. npj Digit. Med. 4, 1-13 (2021).

19. Khera, A. V. et al. Genome-wide polygenic scores for common diseases identify individuals with risk equivalent to monogenic mutations. Nat. Genet. 50, 1219-1224 (2018).

20. Denny, J. \& Basford, M. Type 2 Diabetes - Demonstration Project https://phekb.org/ phenotype/73 (2012).

21. Bielinski, S. J. Heart Failure (HF) with Differentiation between Preserved and Reduced Ejection Fraction https://phekb.org/phenotype/147 (2013).

22. Carlson, C. Dementia https://phekb.org/phenotype/10 (2012).

23. CHOP Phenotyping group, CHOP. Gastroesophageal Reflux Disease (GERD) Phenotype Algorithm https://phekb.org/phenotype/224 (2014).

24. Bycroft, C. et al. The UK Biobank resource with deep phenotyping and genomic data. Nature 562, 203-209 (2018).

25. Wager, S., Wang, S. \& Liang, P. Dropout training as adaptive regularization. Adv. Neural Inf. Process. Syst.26, 351-359 (2013).

26. Green, R. C. et al. ACMG recommendations for reporting of incidental findings in clinical exome and genome sequencing. Genet. Med. 15, 565-574 (2013).

27. Lee, S. et al. Optimal unified approach for rare-variant association testing with application to small-sample case-control whole-exome sequencing studies. Am. J. Hum. Genet. 91, 224-237 (2012).

28. He, Z., Xu, B., Lee, S. \& Ionita-Laza, I. Unified sequence-based association tests allowing for multiple functional annotations and meta-analysis of noncoding variation in metabochip data. Am. J. Hum. Genet. 101, 340-352 (2017).

29. Liu, Y. \& Xie, J. Cauchy combination test: a powerful test with analytic $p$-value calculation under arbitrary dependency structures. J. Am. Stat. Assoc. 115, 393-402 (2020)

30. Karczewski, K. J. et al. The mutational constraint spectrum quantified from variation in 141,456 humans. Nature 581, 434-443 (2020).

31. Wang, K., Li, M. \& Hakonarson, H. ANNOVAR: functional annotation of genetic variants from high-throughput sequencing data. Nucleic Acids Res. 38, e164-e164 (2010).

32. O'Leary, N. A. et al. Reference sequence (RefSeq) database at NCBI: current status, taxonomic expansion, and functional annotation. Nucleic Acids Res. 44, D733-D745 (2016)

33. Adzhubei, I. A. et al. A method and server for predicting damaging missense mutations. Nat. Methods 7, 248-249 (2010)

34. van der Harst, P. \& Verweij, N. Identification of 64 novel genetic loci provides an expanded view on the genetic architecture of coronary artery disease. Circ. Res. 122, 433-443 (2018)

35. Nelson, C. P. et al. Association analyses based on false discovery rate implicate new loci for coronary artery disease. Nat. Genet. 49, 1385 (2017).
36. Klarin, D. et al. Genetics of blood lipids among̃ 300,000 multi-ethnic participants of the million veteran program. Nat. Genet. 50, 1514-1523 (2018).

37. Wojcik, G. L. et al. Genetic analyses of diverse populations improves discovery for complex traits. Nature 570, 514-518 (2019).

38. Peloso, G. M. et al. Association of low-frequency and rare coding-sequence variants with blood lipids and coronary heart disease in 56,000 whites and blacks. Am. J. Hum. Genet. 94, 223-232 (2014).

39. Lange, L. A. et al. Whole-exome sequencing identifies rare and low-frequency coding variants associated with LDL cholesterol. Am. J. Hum. Genet. 94, 233-245 (2014).

40. Do, R. et al. Exome sequencing identifies rare LDLR and APOA5 alleles conferring risk for myocardial infarction. Nature 518, 102-106 (2015).

41. Wahl, P., Ducasa, G. M. \& Fornoni, A. Systemic and renal lipids in kidney disease development and progression. Am. J. Physiol.-Renal Physiol. 310, F433-F445 (2016).

42. Cheedipudi, S. M. et al. Genomic reorganization of lamin-associated domains in cardiac myocytes is associated with differential gene expression and DNA methylation in human dilated cardiomyopathy. Circ. Res. 124, 1198-1213 (2019).

43. Nishiuchi, S. et al. Gene-based risk stratification for cardiac disorders in LMNA mutation carriers. Circulation: Cardiovas. Genet. 10, e001603 (2017).

44. Peretto, G. et al. Cardiac and neuromuscular features of patients with LMNArelated cardiomyopathy. Ann. Intern. Med. 171, 458-463 (2019).

45. Matsuda, T. et al. NF2 activates Hippo signaling and promotes ischemia/reperfusion injury in the heart. Circ. Res. 119, 596-606 (2016)

46. Goek, O.-N. et al. Association of apolipoprotein A1 and B with kidney function and chronic kidney disease in two multiethnic population samples. Nephrol. Dial. Transplant. 27, 2839-2847 (2012).

47. Franceschini, N. et al. The association of cell cycle checkpoint 2 variants and kidney function: findings of the family blood pressure program and the atherosclerosis risk in communities study. Am.J. Hypertens. 22, 552-558 (2009).

48. van der Harst, P. \& Verweij, N. Identification of 64 novel genetic loci provides an expanded view on the genetic architecture of coronary artery disease. Circ. Res. 122, 433-443 (2018).

49. Zhou, W. et al. Scalable generalized linear mixed model for region-based association tests in large biobanks and cohorts. Nat. Genet. 52, 634-639 (2020).

50. Ahuja, Y. et al. sureLDA: a multi-disease automated phenotyping method for the electronic health record. J. Am. Med. Inform. Assoc. 27, 1235-1243 (2020).

51. Aschard, $\mathrm{H}$. et al. Maximizing the power of principal-component analysis of correlated phenotypes in genome-wide association studies. Am. J. Hum. Genet. 94, 662-676 (2014).

52. Liu, Z. \& Lin, X. A geometric perspective on the power of principal component association tests in multiple phenotype studies. J. Am. Stat. Assoc.114, 1-32 (2019).

53. Johnstone, I. M. On the distribution of the largest eigenvalue in principal components analysis. Ann. Stat. 29, 295-327 (2001).

54. Lee, D. D. \& Seung, H. S. Learning the parts of objects by non-negative matrix factorization. Nature 401, 788-791 (1999).

55. Denny, J. C. et al. Systematic comparison of phenome-wide association study of electronic medical record data and genome-wide association study data. Nat. Biotechnol. 31, 1102 (2013).

56. Vilhjálmsson, B. J. et al. Modeling linkage disequilibrium increases accuracy of polygenic risk scores. Am. J. Hum. Genet. 97, 576-592 (2015).

57. Liu, L. \& Kiryluk, K. Genome-wide polygenic risk predictors for kidney disease. Nat. Rev. Nephrol. 14, 723-724 (2018)

58. Khera, A. V. A. Polygenic prediction of weight and obesity trajectories from birth to adulthood. Cell 177, 587-596 (2019).

59. DePristo, M. A. et al. A framework for variation discovery and genotyping using next-generation DNA sequencing data. Nat. Genet. 43, 491 (2011).

\section{ACKNOWLEDGEMENTS}

This research was supported by NIH awards MH106910, MH095797, and U01HG008680. We would like to thank all the investigators and participants of the electronic Medical Records and Genomics (eMERGE) Network. The eMERGE Network was initiated and funded by National Human Genome Research Institute (NHGRI) through the following grants: U01HG006828 (Cincinnati Children's Hospital Medical Center and Boston Children's Hospital); U01HG006830 (Children's Hospital of Philadelphia); U01HG006389 (Essentia Institute of Rural Health, Marshfield Clinic Research Foundation, and Pennsylvania State University); U01HG006382 (Geisinger Clinic); U01HG006375 (Group Health Cooperative and the University of Washington); U01HG006379 (Mayo Clinic); U01HG006380 (Icahn School of Medicine at Mount Sinai); U01HG006388 (Northwestern University); U01HG006378 (Vanderbilt University Medical Center); and U01HG006385 (Vanderbilt University Medical Center serving as the Coordinating Center). This phase of the eMERGE network was initiated and funded by the NHGRI through the following grants: U01HG8657 (Group Health 
Cooperative/University of Washington); U01HG8685 (Brigham and Women's Hospital); U01HG8672 (Vanderbilt University Medical Center); U01HG008666 (Cincinnati Children's Hospital Medical Center); U01HG6379 (Mayo Clinic); U01HG8679 (Geisinger Clinic); U01HG8680 (Columbia University Health Sciences); U01HG8684 (Children's Hospital of Philadelphia); U01HG8673 (Northwestern University); U01HG8701 (Vanderbilt University Medical Center serving as the Coordinating Center); U01HG8676 (Partners Healthcare and the Broad Institute); U54MD007593 (Meharry Translational Research Center); and U01HG8664 (Baylor College of Medicine). The contents of this article are solely the responsibility of the authors and do not necessarily represent the official views of the National Institutes of Health.

\section{AUTHOR CONTRIBUTIONS}

Overall study conceptualization and design (D. X, I. I. L.), algorithm development, testing, software implementation (D. X., I. I. L.), eMERGE data contribution (A. G., I. K., S. M., Y. N., W. W., and C. H. W.), analyses of scores derived from eMERGE phenotype data (D. X., I. I. L., C. W., N. S., C. H. W., and K. K.), genetic association studies with eMERGE-seq panel (D. X., I. I. L., C. W., A. K., Z. H., and K. K.), paper preparation (D. X., I. I. L., C. W., C. H. W., A. G. G., and K. K.). All authors have read and approved the final paper.

\section{COMPETING INTERESTS}

The authors declare no competing interests.

\section{ADDITIONAL INFORMATION}

Supplementary information The online version contains supplementary material available at https://doi.org/10.1038/s41746-021-00488-3.

Correspondence and requests for materials should be addressed to I.I-L.

Reprints and permission information is available at http://www.nature.com/ reprints

Publisher's note Springer Nature remains neutral with regard to jurisdictional claims in published maps and institutional affiliations.

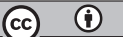

Open Access This article is licensed under a Creative Commons Attribution 4.0 International License, which permits use, sharing, adaptation, distribution and reproduction in any medium or format, as long as you give appropriate credit to the original author(s) and the source, provide a link to the Creative Commons license, and indicate if changes were made. The images or other third party material in this article are included in the article's Creative Commons license, unless indicated otherwise in a credit line to the material. If material is not included in the article's Creative Commons license and your intended use is not permitted by statutory regulation or exceeds the permitted use, you will need to obtain permission directly from the copyright holder. To view a copy of this license, visit http://creativecommons. org/licenses/by/4.0/.

(c) The Author(s) 2021 